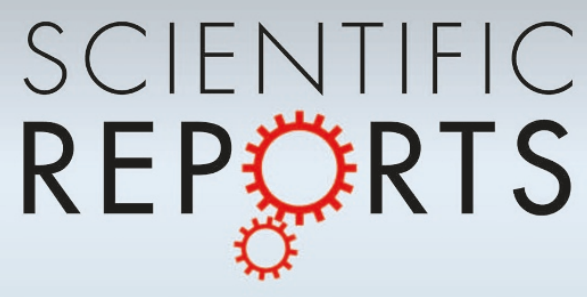

\section{OPEN}

SUBJECT AREAS:

COMPLEX NETWORKS

NONLINEAR PHENOMENA

STATISTICAL PHYSICS

Received

19 November 2013

Accepted

2 June 2014

Published

24 June 2014

Correspondence and requests for materials should be addressed to L.L. (li_lixiang2006@ 163.com)

\title{
Robustness of Interrelated Traffic Networks to Cascading Failures
}

\author{
Zhen Su' ' Lixiang Li', Haipeng Peng ${ }^{2}$, Jürgen Kurths ${ }^{3}$, Jinghua Xiao' \& Yixian Yang ${ }^{2,4}$
}

\begin{abstract}
${ }^{1}$ School of Science, Beijing University of Posts and Telecommunications, Beijing 100876, China, ${ }^{2}$ Information Security Center, State Key Laboratory of Networking and Switching Technology, Beijing University of Posts and Telecommunications, ${ }^{3}$ Potsdam Institute for Climate Impact Research, Potsdam D-14473, Germany, ${ }^{4}$ National Engineering Laboratory for Disaster Backup and Recovery, Beijing University of Posts and Telecommunications, Beijing 100876, China.
\end{abstract}

The vulnerability to real-life networks against small initial attacks has been one of outstanding challenges in the study of interrelated networks. We study cascading failures in two interrelated networks $S$ and $B$ composed from dependency chains and connectivity links respectively. This work proposes a realistic model for cascading failures based on the redistribution of traffic flow. We study the Barabási-Albert networks $(B A)$ and Erdős-Rényi graphs $(E R)$ with such structure, and found that the efficiency sharply decreases with increasing percentages of the dependency nodes for removing a node randomly. Furthermore, we study the robustness of interrelated traffic networks, especially the subway and bus network in Beijing. By analyzing different attacking strategies, we uncover that the efficiency of the city traffic system has a non-equilibrium phase transition at low capacity of the networks. This explains why the pressure of the traffic overload is relaxed by singly increasing the number of small buses during rush hours. We also found that the increment of some buses may release traffic jam caused by removing a node of the bus network randomly if the damage is limited. However, the efficiencies to transfer people flow will sharper increase when the capacity of the subway network $\alpha_{S}>\alpha_{0}$.

M any real-world networks interact with and depend upon other networks ${ }^{1}$. In the past few years, interconnected networks ${ }^{2-4}$ that featured in the interaction among the constituents of a variety of complex systems referred to biological, technological and social systems. The researchers shifted their attention to the multi-layered ${ }^{5-8}$ character of real-world systems that were coupled and especially interdependent with many networks. Real systems are studied to be composed by rather small sub-groups in which the nodes belong to a chain strongly depended on each other'. In our daily life, we experience social behaviors by distinguishing various roles within each single layer we belong to. A network was proposed for two interdependent networks and two types of link containing: connectivity and dependency links ${ }^{10,11}$. Take the city traffic system for example, the subway network and the bus network constitute the daily traffic network, all nodes in different subway lines constitute the subway network, and the nodes of the same subway line depend on each other. Recent studies have shown that the topology of each interdependent layer can affect dramatically the properties of percolation ${ }^{12}$ and cascade failures ${ }^{13}$ in dynamic complex systems. Such systems were found to be more vulnerable compared to classical networks with only connectivity links. Their percolation transition is usually a first-order one compared to the second-order transition found in classical networks ${ }^{14,15}$. City traffic networks as one of such systems, which couple bus and subway networks, has caused much attention ${ }^{16,17}$. Cascading failures are common in most of the coupled communication and transportation networks. The failure of initial removal nodes in one network will cause further failures in other networks and vice versa ${ }^{18}$ resulting in a cascade of failures. Buldyrev et al ${ }^{10}$ studied the robustness of two interacting networks, and found that interdependent networks became significantly more vulnerable compared to their non-interacting counterparts. Cao et al. ${ }^{19,20}$ analyzed the robustness of a network of networks, and gave a general analytical framework for studying percolation of $n$ interdependent networks. It was found by Schneider et al. ${ }^{21}$ that scale-free networks with an onion-like structure are very robust against targeted high degree attacks. An optimal way of interconnection was found against cascading failures ${ }^{22,23}$. Recently, these works of Refs. [9,11,24,25] demonstrated that a crossover in phase transitions of cascading failures can arise in interdependent networks.

The unprecedented pressure of traffic congestion forced many researchers to study the robustness of city traffic networks. Understanding the robustness of city traffic networks has become a major challenge for solving the traffic congestion problem. In previous studies, the failure nodes only cause the failure of nodes that are connected to them directly in one or more networks. The interrelated traffic systems contain dependency subway chains and 


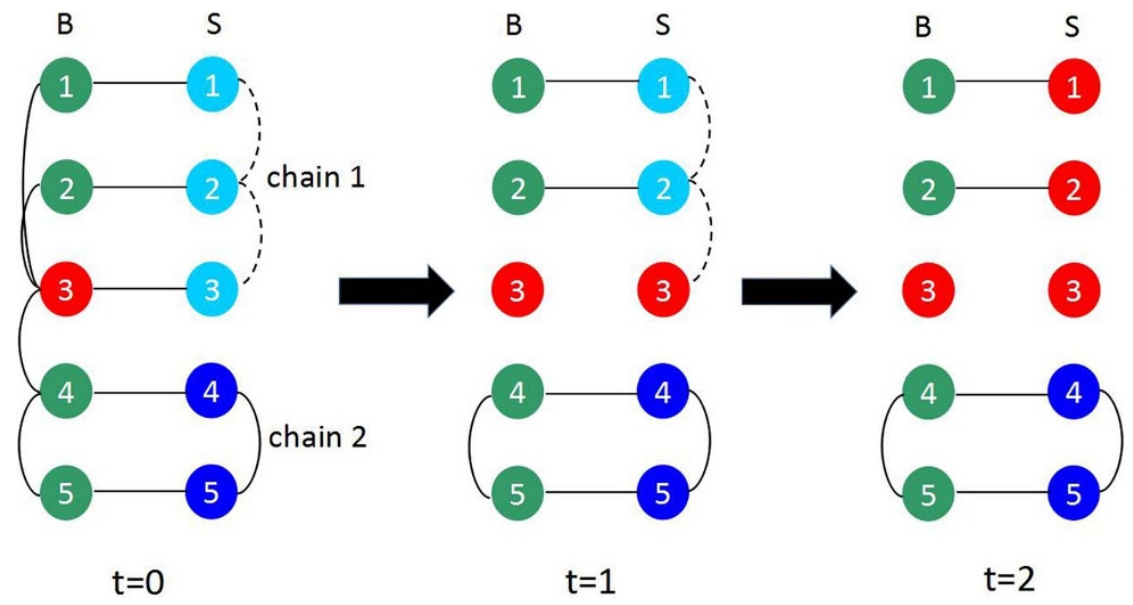

Figure $1 \mid$ Cascading failures in the dependent network $(S)$ and the connected network $(B)$ with the initial removal of a single node chosen at random in the connected network $\boldsymbol{B}$. The colorful solid spheres are different sites. Nodes 1, 2 and 3 linked with broken lines in network $S$ are in the dependent chain 1 . Nodes 4 and 5 being connected with solid lines in network $S$ belong to dependent chain 2 . The nodes with the same number in these two networks are the nodes that have both dependent and connected edges. Suppose that node 3 in network $B$ is attacked at $t=0$, so nodes 3 in network $S$ also could not work at $t=1$. Then nodes 1, 2 and 3 of network $S$ dependent linking with network $S$ could not work in the next time step $t=2$. This may cause the congestion of some relevant nodes in the connected network and an iterative cascading of overload between both networks.

connectivity nodes of buses. A subway network $S$ is composed of nodes, and the subway lines often intersect at the same nodes. The subway network has a special property, i.e. if a node of a dependent subway line (or lines) failed, then it will lead to the failure of its entire dependent nodes of the same line (or lines). That in turn leads to a new failure among connecting nodes of the bus network $B$. Once the cascade process is triggered, it will only stop if nodes that fail in one step do not cause additional failure in the next step. The existing models cannot explain this appropriately. Therefore, to understand the intricate cascade failures of real complex traffic systems where many different attacking rules and load patterns coexist, we need a better model, and a new level of description.

From this perspective, applying real data of Beijing's traffic network, i.e. the subway network and the bus network, we find that the traffic system is robust for the $N R_{B}$ attacking model. Moreover, the efficiency of transferring people flow for removing a node of the bus network randomly $\left(N R_{S}\right)$ is higher with only increasing the tolerance parameter of the subway network $\alpha_{S}>\alpha_{0}$ than that of the bus network $\alpha_{B}>\alpha_{0}$. This means that the increment of some buses may release the traffic jam caused by $N R_{S}$ attacking model if the damage is limited. However, increasing the nodes capacity of the subway network $\alpha_{S}>\alpha_{0}$ will much sharper increase the efficiency to transfer people flow than that of the bus network. It is expected that the proposed model will reflect some important properties of real-life traffic systems when special properties of the subway are taken into account.

Model. To illustrate how the flow redistributes on interrelated networks, we introduce a network model. The network is considered as a weighted and undirected graph $G$ with $N$ nodes. The value of the weighted $\operatorname{arc} e_{i j}$ is a measure of the effect between adjacent nodes $i$ and $j$.

The load $L_{i}$ on node $i$ is the "betweenness" centrality, i.e., the probability of all the shortest paths passing through $\mathrm{it}^{26,27}$. The node capacity $C_{i}$ by using the Motter-Lai model is defined analogously as ${ }^{28}$ :

$$
C_{i}=(1+\alpha) L_{i}, \quad i=1,2, \cdots, N .
$$

The capacity parameter $\alpha \in[0,1]$ is the control parameter representing the tolerance capacity. If one node is removed from the dependency subway network (due to a failure or attack), it affects the shortest paths between some other nodes. Then the loads of many nodes are changed. If it is greater than its capacity, then it will over- load, and the efficiencies of its adjacent edges will decrease. Remote nodes will reselect its shorted path. It triggers further load adjustment over and over again until the loads of all remaining nodes are less than their capacities ${ }^{29}$.

With the above definitions, we can simulate the dynamic process of redistribution of people flows for the initial removal of a node in the related networks. It is well-known that all the sites or nodes on a line cannot work after one interdepended site is destroyed. Without loss of generality, we assume that a node $i$ of the dependent network $S$ is randomly selected as the initial damaged one. So the people at the same line of node $i$ in network $S$ will choose one of the vehicles in the connected network $B$ (for more details, see Fig. 1).

The redistribution rule produce a decrease of the efficiency (refer to Methods section) of the interrelated networks. In order to compare the effects of different attacks for the network's robustness against cascading failures, we adopt the following three basic attacks in the proposed cascading model:

(1) Attack on the nodes randomly (NR): an easy attack strategy, here we compare the result of selecting a node of the dependent $\left(N R_{S}\right)$ and connected $\left(N R_{B}\right)$ network randomly and removing it;

(2) Attack on the node with the highest load $(H L)$ : a common attack strategy, it is to remove the node with the highest load of the system. In heterogeneous networks, e.g., scale-free ones, $H L$ is more likely to trigger cascading failures generally;

(3) Attack on one node of the dependent chain with highest sum loads $(H S L)$ : it is to select the line number of the depended network in ascending order of the sum loads in the depended network and then to remove one node of the chain with the highest loads.

Database in random network and real network. We analyze the robustness of different random networks with connectivity links and dependency chains.

Network models. We study the robustness of $E R$ and $B A$ networks with connected lines and dependency chains. Here we consider two interrelated networks (cf. Fig. 2). Without loss of generality, the initial $E R$ and $B A$ networks are assumed to share the same size initially (i.e., the number of nodes $N=1000$ ). Then choose edges from all possible ones of the $E R$ network with the probability $p^{\prime}=$ 


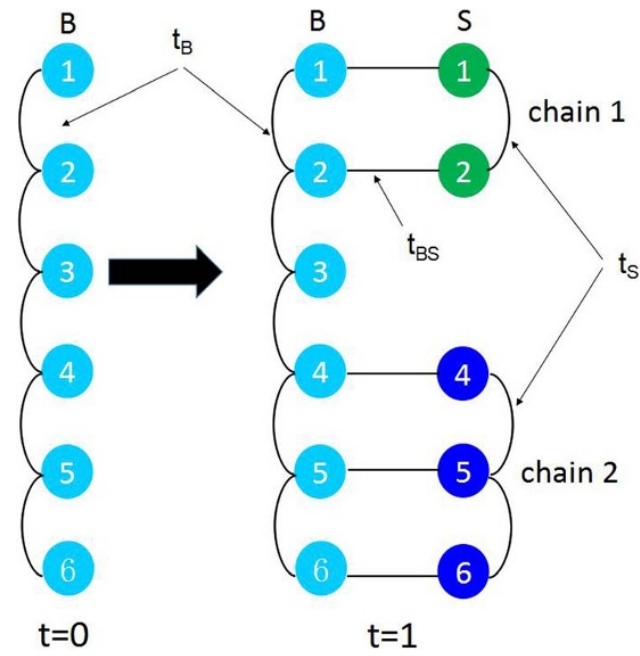

Figure 2 Interrelated random network model with connectivity lines and dependency chains. Suppose that there are six nodes in the initial network $B$ at $t=0$. Select two groups of nodes $\left(B_{1}\right.$ and $B_{2}$ as chain $1 ; B_{4}, B_{5}$ and $B_{6}$ as chain 2$)$ and add their corresponding nodes $\left(S_{1}, S_{2}, S_{4}, S_{5}\right.$ and $\left.S_{6}\right)$ at $t=1$. Here the nodes of $S_{1}, S_{2}, S_{4}, S_{5}$ and $S_{6}$ constitute network $S$. Connect these new nodes of each group with the dependent chains as the new network $S$. Nodes $S_{1}$ and $S_{2}$ are selected to be chain 1 ; nodes $S_{4}, S_{5}$ and $S_{6}$ are selected to be chain 2 . Both networks are coupled by interconnected links of corresponding nodes from both networks, e.g., the $\operatorname{arc}$ of $B_{1}$ and $S_{1}$. $t_{B}, t_{B S}$ and $t_{S}$ are the values of spending time passing two connecting nodes.

0.01; the $B A$ network grows according to the preferential attachment principle that each new node with $m=5$ edges has larger possibility attaching to existing high degree nodes. Then select nodes by the following rule: for a Normal Gaussian distribution with an average

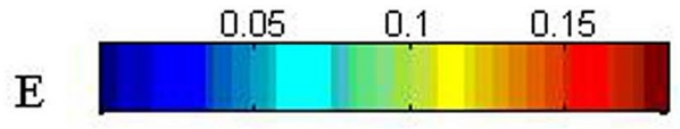

(a)

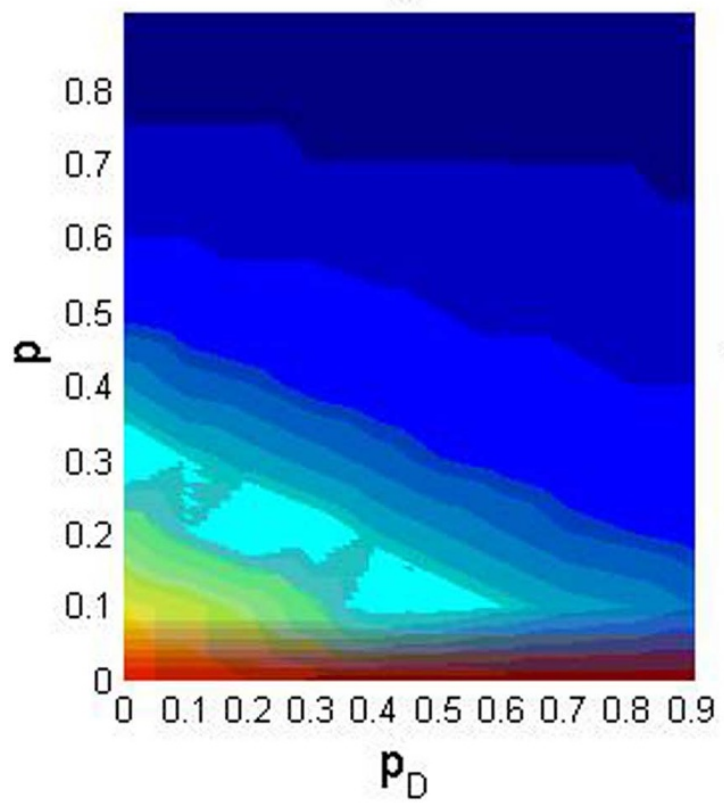

number of nodes $\langle s\rangle=10$ in each dependent chains and the standard deviation $\sigma=0.5$. The initial values of spending time passing two connecting nodes is $t_{B}=2, t_{S}=1$ and $t_{B S}=0.5$ (eq.3).

Real networks. Further we extend the analysis to real systems, the bus and subway networks of Beijing, and check the relationship between $E$ and $\alpha$, which are the average efficiency and the tolerance parameter. We choose all the 227 subway nodes and 924 bus nodes of the lines which have 6 nodes in common with the subway nodes at least, and exert one of the three attack types on the interrelated networks. The largest load node of the systems is on the highest load line. For the NR attacking model, we give the average result over 10 trials.

\section{Results}

In Fig. 3 we find that the efficiencies of both the $E R$ and $B A$ network decrease with the increment of the percentages of dependency nodes $\left(p_{D}\right)$. The efficiency of the ER network is about 0.03 when the percentages of the dependency is 0 and the initial rate of moving nodes is 0.5 . However, for the same efficiency, the initial rate of removing nodes decreases to 0.3 if the percentage of the dependency increases to 0.6. Moreover, $B A$ networks are a little more robust than $E R$ networks with the same increment of $p_{D}$. The efficiencies of $B A \mathrm{~s}$ decrease slower than those of $E R$ networks.

In Fig. 4(a) we show the results of removing a node of the bus network randomly $\left(N R_{B}\right)$ for different values of the tolerance parameter $\alpha$ in Eq.(1). We find that after both systems have reached a stationary state, the average efficiencies of the networks increase for increasing both $\alpha_{S}$ and $\alpha_{B}$. However, the average efficiency of the system with $N R_{B}$ for $\alpha_{S}=0,1, \alpha_{B}=0$ is bigger than that for $\alpha_{S}=$ $0, \alpha_{B}=0.1$. The efficiency of the network is highest for $\alpha_{S}=0.1, \alpha_{B}=$ 0.1 .

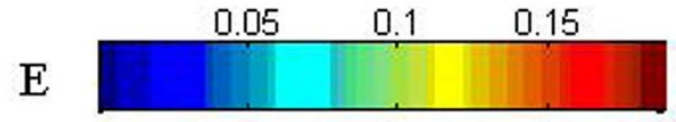

(b)

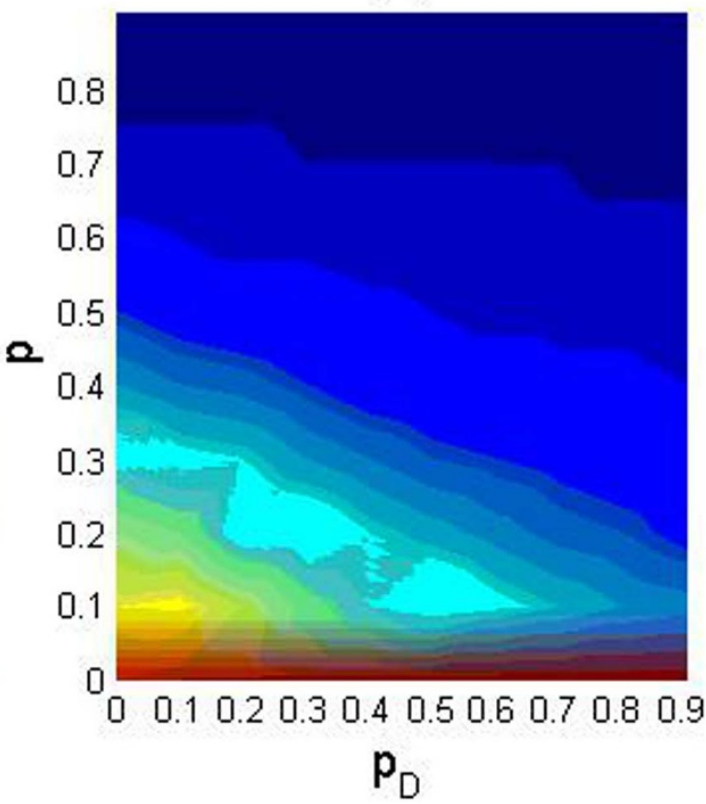

Figure $3 \mid$ Efficiencies (eq.5) of $E R$ and $B A$ networks under randomly removing nodes (NR). $p$ is the initial rate of removing nodes. $p_{D}$ is the percentages of the dependent nodes. (a) Relations of the overload efficiency of the $E R$ network, $p$ and $p_{D}$ with nodes of the network removed randomly. (b) Relations of the overall efficiency of the $B A$ network, $p$ and $p_{D}$ with nodes of the network removed randomly. The value of the tolerance parameter $\alpha$ is 0.03 . Each of the networks has 1000 initial nodes. Each point is from an averaging over 10 realizations. 

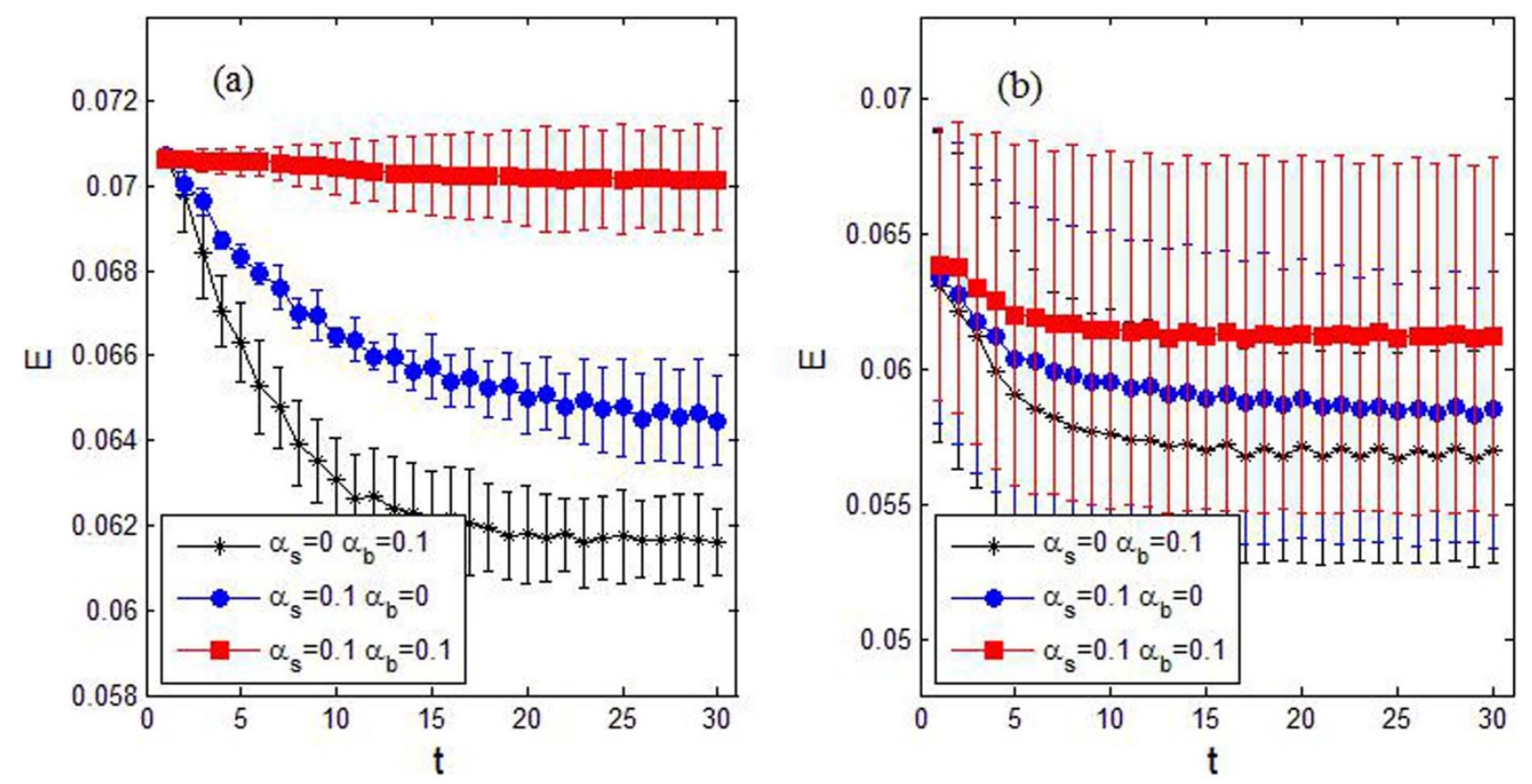

Figure $4 \mid$ Efficiency for the interrelated bus $B$ and subway $S$ network of Beijing. (a) and (b) are the processes of the redistribution of people flows in the interrelated networks with the attacking models of $N R_{B}$ and $N R_{S}$ respectively for different tolerance parameters $\alpha_{S}$ (the capacity parameter of the subway network) and $\alpha_{B}$ (the capacity parameter of the bus network). The efficiency of the system for $\alpha_{B}$ (star) increases $\left(\alpha_{S}=0\right)$ is lower than that as $\alpha_{S}$ (circle) increases $\left(\alpha_{B}=0\right)$ under both attacking models of $N R_{B}$ and $N R_{S}$. The efficiency of the network with $N R_{B}$ attacking model is always greater than that with $N R_{S}$ attack strategy for the same tolerance parameters. For different effects of the dependent chains, there are huge error bars for $N R_{S}$.

We also study the average efficiencies of networks with attacking a node of the subway network randomly $\left(N R_{S}\right)$. In Fig. $4(\mathrm{~b})$ the same situations were found. We also find the efficiency of the networks with $N R_{B}$ attacking model is always higher than that with $N R_{S}$ attack strategy. Huge error bars show there are diverse effects for the system attacking nodes of different dependent chains.

In order to study the influence of the distributed flow on the efficiency of interrelated networks, we compare the efficiencies of these two networks with our four different attacking regimes in Fig. 5(a). We find that the efficiency of the system under the attacking model of $N R_{B}$ is highest. The efficiency under the attacking model of $N R_{S}$ is the next one. For the highest load node belonging to the highest sum loads chain of the subway network, the efficiency of the system under the attacking model of $H L$ is almost equal to that under the attacking model of $H S L$ for each value of $\alpha \in[0,1]$. Both of them are lower than that with $N R_{B}$ and $N R_{S}$ attacking strategies.

We further study the efficiencies of networks under the environment of attacking one node which randomly changes as increasing only the tolerance parameters of the subway network $\left(\alpha_{S}\right)$ or the bus network $\left(\alpha_{B}\right)$ (see Fig. 5(b)). The efficiency of the system with the removal of a node of bus network $\left(N R_{B}\right)$ is higher than that of removing a node of the subway network for each value of $\alpha \in$ $[0,1]$. That is to say, removing a node of the subway network causes more serious damages than that of the bus network. The efficiency of the network as increasing $\alpha_{S}\left(\alpha_{B}=0\right)$ is higher than that increasing $\alpha_{B}$ $\left(\alpha_{S}=0\right)$ for both random removing attacking models $N R_{B}$ and $N R_{S}$. That means the efficiency of increasing the node capacity of the subway network will sharper increase the efficiency of the system than that of increasing the node capacity of the bus network. This confirms the results of Fig. 4.

We further investigate the relationship among the tolerance parameter $\alpha$ and the efficiencies of each network (see Fig. 6). In Fig. 6(a) we can see that the efficiency of the traffic system for $N R_{B}$ attacking model grows quickly to a constant for increasing the tolerance parameter of the subway network $\left(\alpha_{S}\right)$ and the bus network $\left(\alpha_{B}\right)$. However, it grows gradually for $N R_{S}$ (cf. Fig. 6(b)). The overall efficiency of the traffic system under the attacking model of $N R_{B}$ grows rapidly to recover the efficiencies with increasing $\alpha_{B}$ in a small region for some constant $\alpha_{S}$ and vice versa (Fig. 6(c)). So the traffic system is robust for responding to the attacking model of $N R_{B}$. There exists a small critical threshold of the tolerance parameter $\alpha_{0}$ for $N R_{B}$. We find that there exist non-equilibrium phase transitions at the point of $\alpha_{0} \approx 0.1$. The efficiency of the network increases quickly with the increment of the tolerance parameter for $\alpha<\alpha_{0}$, but it will recover immediately when $\alpha>\alpha_{0}$. That is, small increasing of the tolerance parameter of the bus network could decrease the traffic pressure quickly. This explains why the pressure of the traffic congestion is relaxed by increasing some buses during rush hours. In Fig. 6(d), we find that there also exists a small critical threshold of the tolerance parameter $\alpha_{0} \approx 0.1$ for $N R_{S}$. The efficiency of the network for $\alpha_{S}<\alpha_{0}$ and $\alpha_{\mathrm{B}}=0.1$ is lower than that for $\alpha_{\mathrm{B}}<\alpha_{0}$ and $\alpha_{\mathrm{S}}=0.1$. However, it is greater when $\alpha_{\mathrm{S}}>\alpha_{0}$ and $\alpha_{\mathrm{B}}>\alpha_{0}$ respectively. Both efficiencies increase gradually with the increment of the tolerance parameter. So the efficiency of the traffic system recovers gradually as $\alpha_{S}$ and $\alpha_{B}$ increase. Increasing the number of buses can release the traffic jam if it is limited. But the traffic conjunction can be greatly slaked for increasing the tolerance parameter of the subway network $\left(\alpha_{S}\right)$ when $\alpha>\alpha_{0}$ (also could see Fig. 6(d)).

\section{Discussion}

Summing up, we have simulated a flow redistribution model of two types of interrelated networks, i.e. dependent network and connected network, and considered the special property of the subway's structure that all the nodes in the same dependency chain are out of work once one node is failed. Within this framework, we have studied effects of the tolerance parameter of nodes and uncovered that the traffic system of Beijing is robust for an $N R_{B}$ attacking strategy. It will be best to transfer people flow if the tolerance parameter is larger than the critical thresholds. Moreover, we find that the efficiency has a non-equilibrium phase transition at the point of lower $\alpha_{0}$. This implies that increasing the number of small buses can substantially decrease traffic pressure during jam periods. Additionally, the efficiency will be recovered within a very small region of the tolerance parameter $\alpha$. However, the damage of attacking the node of the high- 

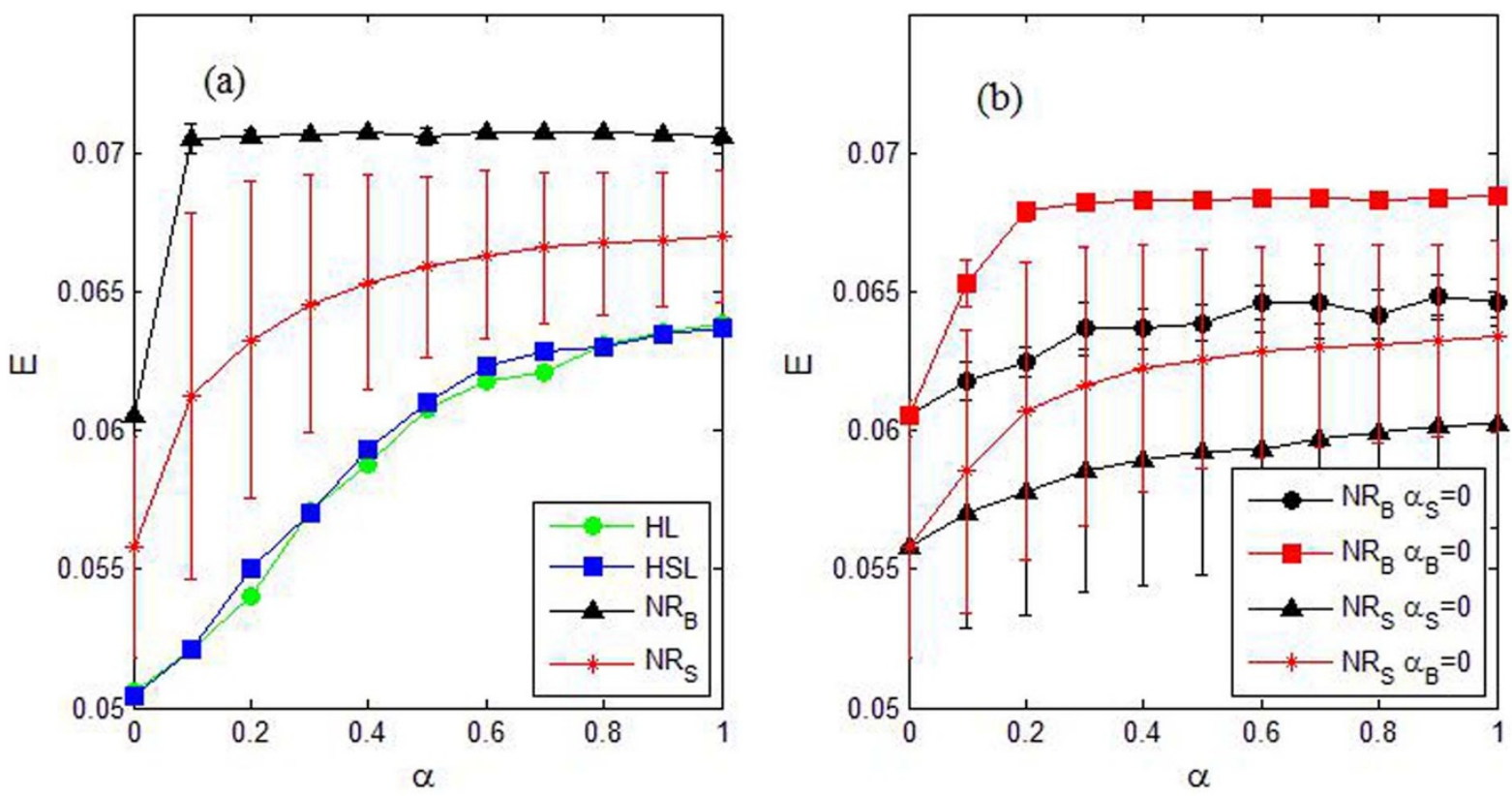

Figure $5 \mid$ (a) and (b) compare the efficiencies of the networks with four different attacking strategies. (a) is the efficiencies of the networks for four different attacking strategies as the tolerance parameters of both bus and subway networks increase at the same time. The green lines (circle) are for the traffic system under the attack of the highest load node $(H L)$; the blue lines (square) are for the traffic system under the attack of the highest sum loads line of the subway network (HSL); the black lines (triangle) are for the traffic system when we remove a node of bus network randomly $\left(N R_{B}\right)$, and the red lines (star) are for the traffic system when we remove a node of subway network randomly $\left(N R_{S}\right)$. The efficiency of the system under the attacking model of $N R_{S}$ as the tolerance parameter increases are highest, following by that under the attacking model of $N R_{S}$. And it is the same lowest with $H L$ and $H S L$.

(b) is to compare the efficiencies with attacking models of $N R_{B}$ and $N R_{S}$ respectively for different tolerance parameters $\alpha$. The red lines (square and star) are for the efficiencies of the networks as increasing the tolerance parameters $\alpha_{S} \in[0,1]$ when $\alpha_{B}=0$. The black lines (circle and triangle) are for the efficiencies of the networks as increasing the tolerance parameters $\alpha_{B} \in[0,1]$ when $\alpha_{S}=0$. All data points of attacking model of $N R$ are averaged over ten times.
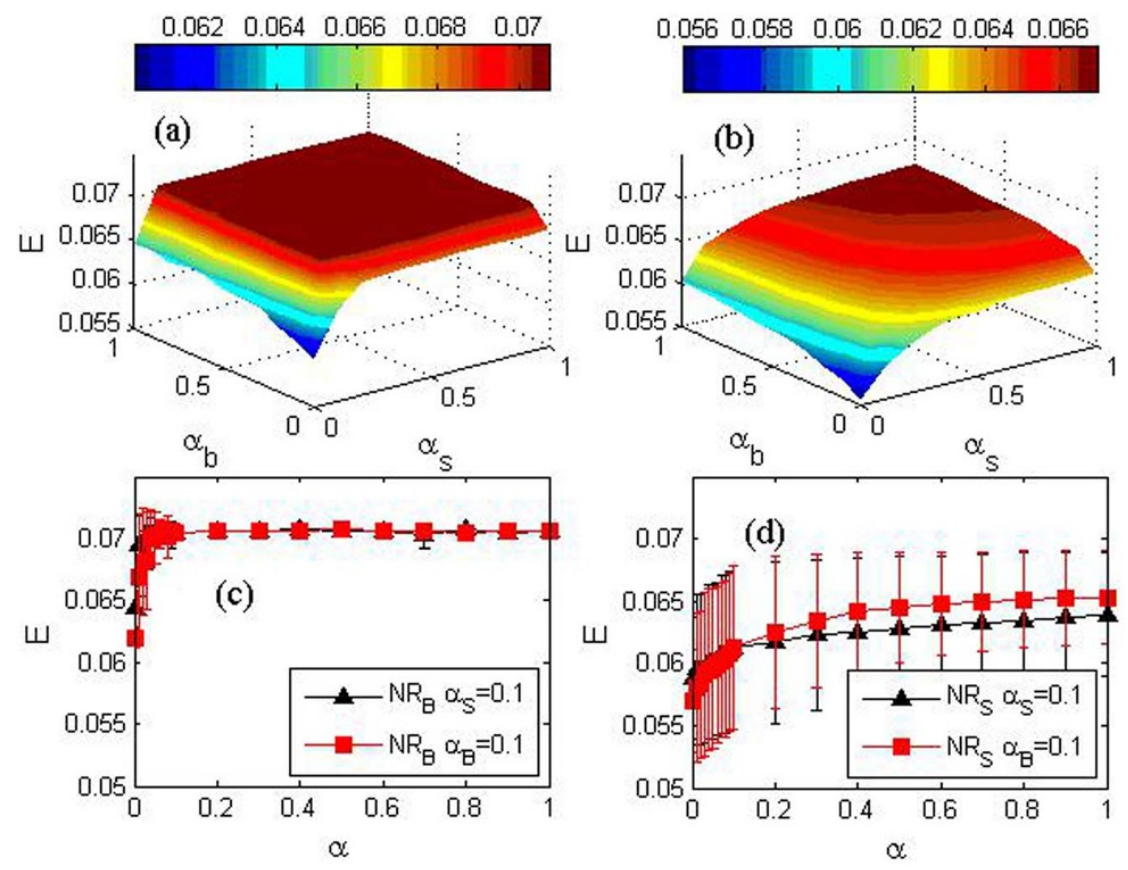

Figure $6 \mid$ In $(\mathrm{a}, \mathrm{b})$, the color 3D curved surfaces are the relations of the efficiency of the traffic system, changing the tolerance parameter of the subway network $\left(\alpha_{S}\right)$ and the bus network $\left(\alpha_{B}\right)$. (a) is the relations of the overall efficiency of the traffic system, $\alpha_{S}$ and $\alpha_{B}$ with a node of the bus network removed randomly $\left(N R_{B}\right)$. (b) is the relations of the efficiency of the subway network subtracting the efficiency of the subway network removed randomly $\left(N R_{S}\right)$. (c) and (d) show some corresponding section planes of (a) and (b), respectively. The black lines (triangles) are the efficiencies of the networks as increasing the tolerance parameter of bus network $\left(\alpha_{B}\right)$ when $\alpha_{S}=0.1$. The red ones (squares) are the efficiencies of the networks as increasing the tolerance parameter of the subway network $\left(\alpha_{S}\right)$ when $\alpha_{B}=0.1$. All the data points are averaged over ten times. 
est load and the node in the highest sum loads line of the subway network is most serious for Beijing's traffic system. Furthermore, increasing the tolerance parameter of the bus network is more effective than that of the subway network for $\alpha<\alpha_{0}$. However, this is contrary to $\alpha<\alpha_{0}$. These findings will be useful for relieving traffic congestion of our daily life. As the proposed model allows for effective simulations, it could also be used for the analysis process of other interrelated networks. Moreover, $B A$ and $E R$ networks with dependency chains and connectivity links are studied. We find that increasing the percentages of dependency nodes could decrease the efficiency of the networks for the NR attacking strategy. The efficiencies of a $B A$ network is a little more robust than that of an $E R$ network with the increment of the percentages of dependency nodes $\left(p_{D}\right)$.

We emphasize the generic character of the cascading failures in Beijing's traffic networks; it can be analogously employed an other city traffic systems with dependency chains and connectivity links, such as Shanghai, Mumbai, Sao Paulo, etc.. Our model is readily applicable to these situations that all the nodes of the same dependency chain will be out of work if one node is attacked. The progress of cascading failures is not limited to assess the effect of overload nodes of city traffic systems, but is also relevant to many aspects of systems with dependency chains and connectivity links. However, this important aspect has received little attention so far.

\section{Methods}

First we show how the damage of a single node (refer to three attacking models in Model section) is sufficient to decrease the efficiency of the entire system simply based on the dynamics of redistribution of the flows on the networks. Suppose a node of network $B$ is attacked at the beginning (see Fig. 1). This leads to changes of the most efficient paths (the shortest ones) between nodes and consequently the loads of nodes in network $B$ redistribute, creating overloads on some nodes. Note that the overloaded nodes are not removed. Instead, for the efficiencies of the arcs, we adopt the following iterative rule at each redistribution time $t>2$ (see Fig. 1):

$$
e_{B_{\mathrm{ij}}}(t+1)=\left\{\begin{array}{cc}
e_{B_{\mathrm{ij}}}(0) & L_{B_{i}}(t) \leq C_{B_{i}} \\
e_{B_{\mathrm{ij}}}(0) \frac{C_{B_{i}}}{L_{B_{i}}(t)} & \text { otherwise }
\end{array}\right.
$$

where it is supposed that the efficiency of the arc is equal to the initial value if the load of node $i$ is not greater than the node capacity $C_{j}$. Otherwise, its efficiency will decrease meaning that the efficiency of all the arcs passing through node $i$ are reduced so that eventually the flow will take alternative paths.

We assume that the spending time passing through the connected nodes $i$ and $j, t_{i j}$, is inversely proportional to the efficiency of the arc, so it is defined by: $t_{i j} \sim \frac{1}{e_{i j}}$. In this paper, we suppose the initial values of spending time passing a subway and bus network arc are $t_{S}=1$ and $t_{B}=2$, respectively. The initial value of time passing between a subway site and a near (less than half distance of two connecting nodes of the bus network) bus site is $t_{B S}=0.5$. We also suppose that all users go along the shortest time path between two sites. Then we have

$$
T_{i j}=t_{i k_{1}}+t_{k_{1} k_{2}}+\cdots+t_{k_{m j}}=\frac{1}{e_{i k_{1}}}+\frac{1}{e_{k_{1} k_{2}}}+\cdots+\frac{1}{e_{k_{m} j}},
$$

where $k_{i}, k_{2}, \ldots k_{m}$ are the nodes on the path from node $i$ to $j$. The efficiency $E_{i j}$ of a path between all vertices $i$ and $j$, can then be defined as the harmonic mean ${ }^{30}$ of the efficiency of the shortest time path between them

$$
E_{i j}=\frac{1}{T_{i j}}=\frac{1}{\frac{1}{e_{i k_{1}}}+\frac{1}{e_{k_{1} k_{2}}}+\cdots+\frac{1}{e_{k_{m} j}}}
$$

The robustness of the whole network is measured by the average transmission efficiency of the network:

$$
E(G)=\frac{1}{N(N-1)} \sum_{i \neq j \in G} E_{i j}=\frac{1}{N(N-1)} \sum_{i \neq j \in G} \frac{1}{\frac{1}{e_{i k_{1}}}+\frac{1}{e_{k_{1} k_{2}}}+\cdots+\frac{1}{e_{k_{m} j}}} .
$$

1. Szabó, G. \& Fáth, G. Evolutionary games on graphs. Phys. Rep. 446, 97-216 (2007).

2. Castellano, C., Fortunato, S. \& Loreto, V. Statistical physics of social dynamics. Rev. Mod. Phys. 81, 591-646 (2009).

3. Barabási, A.-L. The network takeover. Nat. Phys. 8, 14-16 (2012).
4. Zhou, D., Stanley, H. E., D’Agostino, G. \& Scala, A. Assortativity decreases the robustness of interdependent networks. Phys. Rev. E 86, 066103 (2012).

5. Parshani, R., Buldyrev, S. V. \& Havlin, S. Interdependent networks: Reducing the coupling strength leads to a change from a first to second order percolation transition. Phys. Rev. Lett. 105, 048701 (2010).

6. Huang, X., Gao, J., Buldyrev, S. V., Havlin, S. \& Stanley, H. E. Robustness of interdependent networks under targeted attack. Phys. Rev. E 83, 065101(R) (2011).

7. Lee, K.-M., Kim, J. Y., Cho, W.-K., Goh, K.-I. \& Kim, I.-M. Correlated multiplexity and connectivity of multiplex random networks. New J. Phys. 14, 033027 (2012).

8. Peng, H., Wei, N., Li, L., Xie, W. \& Yang, Y. Models and synchronization of timedelayed complex dynamical networks with multi-links based on adaptive control. Phys. Lett. A 374, 2335-2339 (2010).

9. Parshani, R. et al. Critical Effect of Dependency Groups on the Function of Networks. Proc. Nat. Acad. Sci. USA 108, 1007 (2011).

10. Buldyrev, S. V., Parshani, R., Paul, G., Stanley, H. E. \& Havlin, S. Catastrophic cascade of failures in interdependent networks. Nature 464, 08932 (2010).

11. Parshani, R., Buldyrev, S. V. \& Havlin, S. Interdependent Networks Reducing the Coupling Strength Leads to a Change from a First to Second Order Percolation Transition. Phys. Rev. Lett. 105, 048701 (2010).

12. Brummitt, C. D., Lee, K.-M. \& Goh, K.-I. Multiplexity-facilitated cascades in networks. Phys. Rev. E 85, 045102(R) (2012)

13. Cardillo, A. et al. Emergence of network features from multiplexity. Sci. Rep. 3 , 1344 (2013).

14. Tan, F., Xia, Y., Zhang, W. \& Jin, X. Cascading failures of loads in interconnected networks under intentional attack. EPL 102, 28009 (2013).

15. Bashan, A., Parshani, R. \& Havlin, S. Percolation in networks composed of connectivity and dependency links. Phys. Rev. E 83, 051127 (2011).

16. Youn, H., Gastner, M. T. \& Jeong, H. Price of anarchy in transportation networks: efficiency and optimality control. Phys. Rev. Lett. 101, 128701 (2008).

17. Li, G. et al. Towards design principles for optimal transport networks. Phys. Rev. Lett. 104, 018701 (2010).

18. Gao, J., Buldyrev, S. V., Havlin, S. \& Stanley, H. E. Robustness of a network formed by $n$ interdependent networks with a one-to-one correspondence of dependent nodes. Phys. Rev. E 85, 066134 (2012)

19. Gao, J., Buldyrev, S. V., Havlin, S. \& Stanley, H. E. Robustness of a Network of Networks. Phys. Rev. Lett. 107, 195701 (2011).

20. Gao, J., Buldyrev, S. V., Havlin, S. \& Stanley, H. E. Networks formed from interdependent networks. Nat. Phys. 8, 40 (2012).

21. Schneider, C. M., Araújo, N. A. M., Moreira, A. A., Havlin, S. \& Herrmann, H. J. Mitigation of malicious attacks on networks. Proc. Nat. Acad. Sci. USA 108, 3838 (2011).

22. Schneider, C. M. et al. Suppressing Cascades of Load in Interdependent Networks. Proc. Nat. Acad. Sci. USA 109, E680 (2012).

23. Liu, G. L., Peng, H. P., Li, L. X., Sun, F. \& Yang, Y. X. Improving resource utilization in hierarchy network by optimizing topological structure. Eur. Phys. J. $B$ 85, 63 (2012).

24. Gómez, S. et al. Diffusion Dynamics on Multiplex Networks. Phys. Rev. Lett. 110, 028701 (2013).

25. Hu, Y., Ksherim, B., Cohen, R. \& Havlin, S. Percolation in Interdependent and Interconnected Networks: Abrupt Change from Second- to First-order Transitions. Phys. Rev. E 84, 066116 (2011).

26. Goh, K.-I., Kahng, B. \& Kim, D. Universal Behavior of Load Distribution in ScaleFree Networks. Phys, Rev. Lett. 87, 278701 (2001).

27. Latora, V. \& Marchiori, M. Efficient Behavior of Small-World Networks. Phys, Rev. Lett. 87, 198701 (2001)

28. Motter, A. E. \& Lai, Y. C. Cascade-Based Attacks on Complex Networks. Phys. Rev. E 66, 065102(R) (2002).

29. Morris, R. G. \& Barthélemy, M. Transport on coupled spatial networks. Phys. Rev. Lett. 109,128703 (2012).

30. Crucitti, P., Latora, V. \& Marchiori, M. Model for Cascading Failures in Complex Networks. Phys. Rev. E 69, 045104(R) (2004).

\section{Acknowledgments}

This work was partly supported by the National Natural Science Foundation of China (Grant Nos. 61100204, 61170269, 61121061), the Asia Foresight Program under NSFC Grant (Grant No. 61161140320), the Beijing Natural Science Foundation (Grant No. 4142016) and the Beijing Higher Education Young Elite Teacher Project (Grant No. YETP0449), the Beijing Center for Mathematics and Information Interdisciplinary Sciences, and by the Government of the Russian Federation (Agreement No. 14.Z50.31.0033)

\section{Author contributions}

Z.S. and H.P. designed research; Z.S., H.P., L.L. and J.K. performed research; Z.S., J.X., L.L. and Y.Y. explained the results; Z.S., J.K., J.X. and Y.Y. wrote and corrected the paper.

\section{Additional information}

Competing financial interests: The authors declare no competing financial interests.

How to cite this article: Su, Z. et al. Robustness of Interrelated Traffic Networks to Cascading Failures. Sci. Rep. 4, 5413; DOI:10.1038/srep05413 (2014). 
cc)(i) (2) This work is licensed under a Creative Commons Attribution-NonCommercialShareAlike 4.0 International License. The images or other third party material in this article are included in the article's Creative Commons license, unless indicated otherwise in the credit line; if the material is not included under the Creative
Commons license, users will need to obtain permission from the license holder in order to reproduce the material. To view a copy of this license, visit http:// creativecommons.org/licenses/by-nc-sa/4.0/ 